\section{6}

\section{Hewlett-Packard}

\section{Europhysics} Prize

The EPS Hewlett-Packard Europhysics prize awarded each year by the European Physical Society for outstanding achievement in solid state physics has been awarded this year to Professor Dr. Wolfgang Helfrich for his contributions to the physics of liquid crystals, leading also to the discovery of the twisted nematic display and to a better understanding of the behaviour of cerlain biological phenomena. Professor Helfrich's largely theoretical work explained how some fibrelike (nematic) molecules, change their optical properties when a voltage is applied across them, both in the case of current flow, when instabilites appear, and in the case of no current flow where no power is consumed. A few volts can change the liquid crystal from transparent to opaque.

Professor Helfrich is now at the Freie Universität in Berlin. $\mathrm{He}$ carried out the research for which the award is made between 1969 and 1972 when at the RCA Laboratories in Princeton, USA and at F. Hoffman-La Roche in Basle, Switzerland.

The EPS-H.P. prize is donated by Hewlett-Packard and is worth $20000 \mathrm{Sw}$. Frs. It will be presented to Professor Helfrich at the opening of the 19th Congress Ampere at Heidelberg on 27 September 1976.

\title{
Fourth General Conference of the European Physical Society
}

As was decided by Council at its meeting in Leipzig, the next general conference of the European Physical Society - the fourth of the series will take place at the University of York, in the United Kingdom, from 25 to 29 September 1978.

The conference will extend over five days and include plenary sessions and several symposia in five parallel sessions. Time will also be devoted to the General Assembly of EPS members and to the Powell Memorial Lecture.

The various symposia will be organized under the responsibility of the EPS Divisions and ACAPPI. The topics chosen - which will now have to be more sharply defined - are listed below. It will be seen that preference has been given in the choice of the topics to interdisciplinary interdivisional subjects.

\section{The Five Parallel Sessions :}

A Modern Optics

B1 Surface Physics

B2 Theoretical Methods for Phase Transitions

C1 Nuclear Astrophysics

C2 Hot Plasmas

D Heavy Ion Physics

E1 Synchrotron Radiation and its Application

E2 A topic to be decided by the High Energy and Particle Physics Division (possibly Quarks).

Some of the symposia should include contributed papers, the other symposia might consist only of invited and review (rapporteur) papers.
The choice of the talks in the plenary sessions for which several proposals have already been received, will be made in 1977. Physics and Society and Physics Education will also be included in the programme.

There will be a general international programme committee for the whole conference; each of the symposia will be organized by a small scientific committee consisting of representatives of the various divisions and of the Advisory Committee involved.

\section{Europhysics Conferences}

Rules governing conferences eligible for EPS sponsorship and the title Europhysics Conference were first published in Europhysics News $\mathbf{3}$ No. 5, June 1972. Subsequent amendments were announced in EN 5, No. 5, May 1974 ; 5, No. 11, Nov. 1974 and 6, No. 5, Summer 1975.

Enquiries about EPS approval or sponsorship and requests for the complete guide, together with amendments, should be made to the EPS Secretariat.

\section{EPS Holidays}

Readers should note that the EPS Secretariat will be closed for the Summer Holidays from 19 July to 9 August. In case of real urgency the secretary can be reached on Geneva (22) 345216. 


\section{Guide to Entries}

This Meetings Issue of Europhysics News, contains details of future meetings about which notification was received at the EPS Secretariat in Geneva by May 1976. The events are listed in date order in two categories - Conferences and Schools. Europhysics Conferences, i.e. meetings Sections are printed in bold type and boxed ; events sponsored by EPS are printed in bold type; Europhysics Study Conferences are marked by an $\Theta$ preceding the date.

Conference and school organizers who wish to have their meetings included in the next Meetings Issue of Europhysics News should apply to: European Physical Society,

P.O. Box 39 ,

$\mathrm{CH}-1213$ Petit-Lancy 2

In general, meetings are open to all applicants; where attendance is known to be restricted to invited applicants, this is indicated by 'inv.'. The information is given in the following order :

\section{Date}

Venue

Title

Contact for information

A: Application deadline / Ab: Abstracts deadline / P: Papers deadline / Number of participants / inv.: by invitation only.

Languages other than English / Sponsorship / Conference fee.

An asterisk (*) preceding the Title indicates that full information can be found in the Meetings Issue of March 1976 (Vol. 7, No. 3). organized by EPS, its Divisions and

\section{Conferences}

\section{6}

9-13 July

Stockholm, Sweden

* Symposium on Antinucleon-Nucleon Reactions

12-16 July Bristol, UK

* 3rd Int. Conf. on the Properties of Liquid Metals

\section{2-16 July}

Oxford, UK

E Low Temperature Nuclear Orientation N.J. Stone, Clarendon Laboratory, Parks Road, Oxford OX1 $3 \mathrm{PU}$

A: $1.5 .76 / 70$

f 55.- (all incl.)

13-16 July

8th EGAS Conference on Atomic Spectroscopy

D.N. Stacey, Clarendon Laboratory, 200 Parks Road, Oxford OX1 3PU

15-21 July

* 18th Int

Physics

19-20 July

Reading, UK

Photoelectron Spectroscopy

The Meetings Officer. The Institute of Physics, 47, Belgrave Square, London SW1X $8 Q X$ $\mathrm{Ab}: 20.5 .76$

20-23 July Nottingham, UK

Probability in Quantum Theory

R.L. Hudson, Mathematics Dept., University of Nottingham, University Park. Nottingham NG7 2RD Ottawa, Ontario, Canada * 4th International Conf. on Medical Physics

26-30 July Berkeley, Cal., USA

R. Marrus, Dept. of Physics, University of California, Berkeley, Cal. IUPAP

1-7 Aug.

Toronto, Canada

12th Int. Congress on High Speed Photography Congress Secretariat, c/o Ontario Science Centre, 770 Don Mills Road Don Mills, Ontario, Canada M3C 1T3 5th Int. Conf. on Atomic Physics

\section{Energy and Physics}

\section{Published by European Physical Society, P.O. Box 39 CH-1213 Petit-Lancy 2, Switzerland}

Please enter my order and send me your invoice

Name (in CAPITALS)

\section{Address :}

Order No.

Place, Date

Signature

Ind. Ord. Member No.

Participant in the Conference

YES
10-12 Aug.

Gothenburg, Sweden

Nordic Laser and Electro-Optics Conference NOLEC '76

Sverre T. Eng, Chairman, Dept. of Electrical Measurements, Chalmers University of

Technology, Fack, S-402 20 Gothenburg A: $1.6 .76 / 50 /$ inv

sKr. 600 .-

16-20 Aug.

Nice, France

* Problems of Stellar Convection

16-20 Aug.

Cambridge, UK

* Radio Astronomy and Cosmology

16-20 Aug

Kiljava, Finland

* SAGAMORE V: 5 th Conf. on Charge Spin and Momentum Density

16-20 Aug.

Sart-Tilman, Liège, B

17th Session, Int. Post-University Courses

P.O. Box 24, B-1000 Brussels 29

A: $31.7 .76 / 300$

English, French, German/bFr. 200.-

17-19 Aug.

Cambridge, UK

* Dynamics of Planets and Satellites and

Theories of their Motion

17-20 Aug.

Palo Alto, Cal., USA

* Applied Superconductivity Conference

17-20 Aug.

Lyons, France

* Relationship between Comets, Minor Planets and Meteorites

17-21 Aug Novosibirsk, USSR

* 7th International Symposium on Discharges and Electrical Insulation in Vacuum

19-21 Aug.

Strasbourg. France

* The Compilation, Critical Evaluation and

Distribution of Stellar Data

19-28 Aug. Garmisch-Partenkirchen, FRG * Radiation in the Atmosphere - Structure and Radiation Properties of Aerosols and Clouds

23-26 Aug. Helsingor, Denmark * 4th Int. Conf. on Positron Annihilation

23-26 Aug. Jerusalem, Israel Int. Conf. on Holography and Optical Data Processing

E. Marom, School of Engineering,

Tel-Aviv University, Ramat-Aviv

A: $30.6 .76 / \mathrm{Ab}: 15.3 .76 / \mathrm{P}: 23.8 .76 / 250$

US $\$ 60$. - ; after 30.6 .76 US $\$ 70$.-

23-27 Aug.

Gothenburg. Sweden

7th International Congress on Rheology

Gothenburg Convention Bureau, Parkgatan 2, S-411 38 Gothenburg

A: $1.4 .76 / P: 15.2 .76$

sKr. 300.-

23-27 Aug.

Argonne, III., USA

3rd Argonne Summer Symposium: High Energy Physics with Polarized Beams and Targets

J.L. Marshak, School of Physics and Astronomy, University of Minnesota, Minneapolis, MN 55455

23-27 Aug.

Kent, Ohio, USA

* 6th International Liquid Crystal Conference

23-28 Aug. Delft, The Netherlands

8th AICA Congress - Simulation of Systems and 5th Int. Colloquium on the Beuken-Model Secretariat 8th AICA Congress, Computer Centre, Delft University of Technology, Michiel de Ruyterweg 10-12, P.O.B. 354, Delft

A: 15.6 .76

hFl. 300.

24-27 Aug. Bratislava, Czechoslovakia 3rd European Study Conf. on Atomic and Molecular Physics of Ionized Gases ESCAMPIG

P. Lukac, Katedra esperimentalnej fyziky PFUK, Mlynska dolina F 1, 81631 Bratislava

Ab: 1.4 .76

US \$ 45.- 
24-31 Aug.

Technion City, Haifa, Israel

* Haifa International Workshop: Topics in Atomic Physics related to Nuclear Experimentation

24 Aug. - 2 Sept.

* IAU General Assembly

Grenoble, France

30 Aug. - 1 Sept.

Amsterdam, NL

Exploring the Chemical Bond,

New Developments

Miss A.-M. Bonino, Battelle Institute, Advanced Studies Center

7, route de Drize,

CH-1227 Carouge-GE, Switzerland

A: $1.5 .76 /$ Ab: $1.6 .76 /$ P: $1.8 .76 / 150$

hFI. 120.-

30 Aug. - 1 Sept.

Stockholm, Sweden

Long-Term Properties of Polymers and Polymeric Materials

B. Ranby, Department of Polymer Technology, Royal Institute of Technology.

S-100 33 Stockholm

30 Aug. - 2 Sept. Gaithersburg, Md., USA

* Int. Conf. on the Physics of X-Ray Spectra

30 Aug. - 3 Sept.

Berlin-West

Lattice Defects in Ionic Crystals

F.W. Felix, Hahn-Meitner-Institut für Kernforschung Berlin GmbH, Dept. C-d, Glienicker Str. 100, D-1000 Berlin-39

A: 31.5.76 / Ab: 28.2.76 / P: 28.2.76 / 250

DM 75.-

30 Aug. - 3 Sept.

Geneva, Switzerland

* Workshop on Problems Related to Solar Energy Utilization

30 Aug. - 3 Sept.

Nancy, France

* 4th Int. Conf, on Strength of Metals and Alloys

30 Aug. - 3 Sept.

Rome, Italy

* 13th Int. Conf. on the Physics of Semiconductors

30 Aug. - 4 Sept.

Delft, The Netherlands

* 14th Int. Congress of Theoretical and Applied Mechanics

30 Aug. - 4 Sept.

Dubrovnik, YU

9th Int. Seminar of Int. Centre for Heat and Mass Transfer

International Centre for Heat and Mass Transfer, P.O. Box 522, YU-1100 Belgrade

A: $1.8 .76 / 200$

US $\$ 150,-$ (incl. proceedings)

31 Aug. - 3 Sept.

Uppsala, Sweden

Vacuum and Thin Film Technology

Peter Andersson, Institute of Technology, Box 534, S-751 21 Uppsala

A: 1.3.76 / Ab: 1.4 .76 / P: $1.5 .76 / 250$

sKr. 300 .-

1-3 Sept. London, UK

* Advances in Magnetic Materials and their Applications

1-3 Sept.

Zurich, Switzerland

Magnetooptics

P. Wachter, Laboratorium für Festkörperphysik, ETH Hönggerberg, CH-8049 Zurich

A: 1.6.76 / Ab: 1.4.76 / P:1.8.76 / 150

IUPAP / SFr. 150.-

1-3 Sept.

Lisbon, Portugal

* 3rd Int. Conf. on Electrode Phenomena in Gas Discharges

1-4 Sept.

Zurich, Switzerland

Crystal Field Effects in Metal and Alloys

5-11 Sept.

Trieste, Italy

* Physics and Astrophysics from Spacelab

6-7 Sept.

Paris, France

* Redshif

Universe

6-8 Sept. Meudon, France

* Astronomical Application of Image Detectors wth Linear Response

6-8 Sept.

Dublin, Ireland

* 14th Annual Meeting of European High Pressure Research Group - High Pressure Techniques for Science and Technology

6-9 Sept. Dubrovnik, Yugoslavia * Radiation Effects in Semiconductors

6-9 Sept. Nice, France

* Energy Balance and Hydrodynamics of the Chromosphere and Corona

6-10 Sept. Amsterdam, The Netherlands * International Conference on Magnetism. (ICM 76)

\section{6-10 Sept. \\ Nuclear Physics with Heavy lons \\ Mme M.S. Detœuf, IN2P3, 11, rue Pierre et Marie Curie, F-75231 Paris Cedex 05 A: $15.10 .75 / 300$}

6-10 Sept.

Geneva, Switzerland

Star Formation

G.B. Field, Center for Astrophysics, 60 Garden Street, Cambridge, Mass. 02138, USA

$100 /$ inv.

English, French / IAU

6-10 Sept.

Zurich, Switzerland

* 3rd European Crystallographic Meeting

6-10 Sept. Bari, Italy

* Layered Semiconductors and Metals

6-11 Sept.

Montpellier, France

* 3rd IUPAC Conf. on Physical Organic Chemistry

7-9 Sept.

Swansea, UK

* Gas Discharges

7-9 Sept.

* Novae and Related Stars

Paris, France

7-10 Sept.

Genoa, Italy

* European Conf. on Circuit Theory and Design

7-10 Sept.

Amsterdam, NL

* Waves in Upper Atmosphere: Magnetopause Regions ; Interplanetary Medium

8-9 Sept.

Paris, France

* L'Evolution des Galaxies et ses Implications Cosmologiques

8-9 Sept.

Nemu-no-sato, Mie, Japan

* IEE Specialists Conf.: Technology of Electroluminescent Diodes

8-10 Sept.

Potential Energy Surfaces

Brighton, UK

Mrs. Y.A. Fish, Faraday Division, The Chemical Society, Burlington House, London W1V OBN A: $9.8 .76 / P: 3.5 .76 / 150 /$ inv.

\& 12 -
10-12 Sept.

Worcester, UK

Schol Phys for the 1980's

The Meetings Officer, The Institute of Physics,

47. Belgrave Square, London SW1X $8 Q X$

12-18 Sept.

Zurich, Switzerland

* 1st European Conf. on Crystal Growth (ECCG-1)

13-15 Sept.

Itinerant Electron Magnetism

Oxford, UK

E.P. Wohlfarth, Department of Mathematics,

Imperial College of Science and Technology,

Exhibition Road, London SW7 2RH

A: May $76 / 150 /$ inv.

\& 15.-

13-16 Sept.

Eindhoven, NL

* 2nd International Conf. on Magnetic Bubbles

13-16 Sept.

Noordwijk, NL

Int. Study Conf. on Photo Emission from Surfaces R.F. Willis, Astronomy Division, ESTEC, inv. Noordwijk

13-16 Sept.

Nijmegen, NL

* Phonons, Soft Modes and Superconductivity

13-16 Sept.

Wroclaw, Poland

* 2nd Int. Conf. on the Electronic Structure of the Actinides

13-17 Sept.

Munich, FRG

6th European Solid State Device Research Conference (ESSDERC 1976)

R. Müller, Technische Universität, Lehrstuhl für Technische Elektronik, Arcisstrasse 21, D-8 Munich 2

A: $\mathbf{5 . 5 . 7 6 / P : ~} \mathbf{3 0 . 6 . 7 6}$

DM 150.-

13-17 Sept.

Corfu, Greece

* Int. Conf. on Applications of Mössbauer Effect

13-17 Sept.

Small Particles and Inorganic Clusters

B. Cabaud, Département de Physique des

Matériaux, 43, bd du 11 Novembre 1918

F-69621 Villeurbanne

A: $30.5 .76 / 100$

English, French / FF 300.-

13-17 Sept.

London, UK

3rd Int. Conf. on Spectral Line Shapes

The Meetings Officer. The Institute of Physics,

47. Belgrave Square, London SW1X $8 \mathrm{QX}$

Ab: 1.6 .76

14-16 Sept.

Capodimonte Obs., Italy

Two-Dimensional Radiative Transfer - Theory and Application to Structures in Stellar Atmospheres

B. Caccin, Osservatorio Astronomico Capodimonte P.G., Via Moiariello 16, 1-80131 Naples $20 /$ inv

14-17 Sept.

Bonn, FRG

40th General Meeting of German Physical Society Deutsche Physikalische Gesellschaft e.V., Hauptgeschäftsstelle, Gotenstrasse 1-3,

D-5300 Bonn-Bad Godesberg 1

14-17 Sept.

Leeds, UK

European Cosmic Ray Symposium

C.J. Hatton, Physics Dept., University of Leeds, Leeds LS2 9JT, UK

A: $1.4 .76 /$ Ab: $1.6 .76 / 120$

\& 7.50

* For full details please see March issue of Europhysics News 
14-17 Sept.

Bellevue, France

2nd Int. Conf. on Ferrites - ICF 2

A. Globus and V. Cagan, CNRS, F-92190 Bellevue

Ab: 1.5.76 / P: 1.5.76/150/Inv.

English, French / IUPAP / FF 300.- (IOM, members adh. Soc. EPS), FF 330.- (others)

14-17 Sept.

Chalk River, Canada

* 1976 Proton Linear Accelerator Conference

\section{4-17 Sept.}

Rome, Italy

* 6th European Microwave Conference

14-20 Sept.

Jerusalem, Israel

6th European Congress on Electron Microscopy Secretariat, 1976 European Conf. on Electron Microscopy, POB 16271, Tel-Aviv

$A b: 15.3 .76$

15-18 Sept.

York, UK

8th Int. Colloquium on Magnetic Thin Films

M. Prutton, Physics Department, University of York, Heslington, York YO1 5DD

$\mathrm{Ab}: 1.6 .76$

17-18 Sept.

Glasgow, UK

* Thermal Acoustic and Viscoelastic Properties of Polymers

17-20 Sept.

* Experimental Gravitation

Pavia, Italy

19-22 Sept.

Edinburgh, UK

* Symposium on Gallium Arsenide and

Compounds

20-23 Sept.

* Akustik (DAGA '76)

Heidelberg, FRG

20-23 Sept.

Cambridge, UK

* Int. Conf. on Structure of Noncrystalline Materials

20-24 Sept.

London, UK

* International Broadcasting Convention (IBC 76)

21-23 Sept.

York, UK

* Optical Group Conference 1976 (IOP)

21-24 Sept.

Toulouse, France

2nd European Solid State Circuits Conference (ESSCIRC 76)

ESSCIRC 76, LAAS-CNRS, 7, avenue du ColonelRoche, F-31400 Toulouse

A: July 76 / Ab: 5.5.76/ P: 5.5.76/500

English / FF 500.-

21-24 Sept.

Liège, Belgium

Workshop: Electronic Structure and Phase Stability in Metals and Alloys

R. Evrard, Université de Liège, Institut de Physique, B-4000 Sart Tilman par Liège 1 bFr. 2500 .-

\section{2-24 Sept.}

York, UK

Coherent Laser and Microwave Spectroscopy

Dr. J.G. Baker, Schuster Laboratory, University, Manchester M13 9PL

A: $4.9 .76 / \mathrm{P}: 16.8 .76 / 100$

22-24 Sept.

Liverpool, UK

* Microanalytical Techniques
25-28 Sept.
Lancaster, UK
G Dilution Refrigeration (Study Conference)
Miss C.J. Martin, Physics Dept., University of Lancaster, Lancaster LA1 4YB
A: $24.8 .76 / 100 /$ inv.
\& 46 (all incl.)

25-29 Sept.

Sunny Beach, Bulgaria

* VII. Nat. Conf. on Spectroscopy (with international participation)

27-30 Sept.

Linz, Austria

Annual Meeting of the Austrian Physical Society Johannes Kepler University, OPG Tagung 1976 A-4045 Linz

27-30 Sept.

Paris, France

* 2e Colloque Européen sur les Transmissions par Fibres Optiques

27 Sept. - 1 Oct.

Heidelberg, FRG

19th Ampère Congress on Magnetic Resonances

H. Brunner, Secretary, 19th Ampère Congress, Max-Planck-Institut, Abt. Molekulare Physik, Jahnstrasse 29, D-6900 Heidelberg

A: $15.6 .76 /$ P: $15.6 .76 / 600$

English, French, German / IUPAP / US \$ 60.-

30 Sept. -6 Oct. Munich, FRG * 9th Int. Conf. on Solid State Nuclear Track Detectors

1 Oct.

London, UK

Instrumental Developments in Surface Spectroscopy

The Meetings Officer, The Institute of Physics, 47, Belgrave Square, London SW1X $8 Q X$

4-5 Oct.

London, UK

* The Operation of Instruments in Adverse Environments

5-8 Oct.

Geneva, Switzerland

Electro-Optics and Interactive Computers Graphics Conference and Exhibition

Information-Network, 84 High Street, Newport Pagnell, Bucks., MK16 8EG, UK

5-8 Oct.

Chicago, III., USA

Fast Reactor Safety and Related Physics

Robert Avery, Argonne National Laboratory, BIdg. 208, Argonne, III. 60439

Ab: 5.4 .76

5-8 Oct.

Berlin-West

SQUIDS - Int. Conf. on Superconductng Quantum Devices

H.D. Hahlbohm, Physikalisch - Technische Bundesanstalt, Institut Berlin, Abbestrasse 2-12, D-1 Berlin 10

A: $5.10 .76 / P: 1.7 .76 / 150 /$ inv.

English / US \$ 50.-

6-7 Oct.

Washington, D.C., USA

The Importance of Scientific and Technical Information

ICSU AB, 17 rue Mirabeau, F-75016 Paris

6-8 Oct.

Upton, L.I., USA

Meeting of the Particles and Fields Division (APS) American Physical Society, 335 E 45th Street, New York, N.Y. 10017

6-13 Oct.

Berchtesgaden, FRG

6th Conference on Plasma Physics and Controlled Nuclear Fusion Research

Conference Service, IAEA, POB 590, A-1011 Vienna

10-15 Oct.

Amherst, Mass., USA

* Int. IEEE/AP Symposium and USNC/URSI Meeting

11-15 Oct.

Liège, Belgium

* 8th International Congress on Electro-Heat

12-14 Oct.

Seattle, Wash., USA

8th National SAMPE Technical Conference: Bicentennial Materials Review - Part II SAMPE, P.O. Box 613, Azusa, Cal. 91702
12-15 Oct.

Saclay, France

* Electrical Phenomena at Membrane Level

18-22 Oct.

Argonne, III., USA

* Properties of Atomic Defects in Metals

18-22 Oct.

Jülich, FRG

Int. Symposium on Plasma Wall Interaction

H. Gresser, Institut für Plasmaphysik,

Kernforschungsanlage Jülich $\mathrm{GmbH}$,

P.O. Box 1913, D-5170 Jülich

A: $1.7 .76 / \mathrm{Ab}: 15.8 .76 / \mathrm{P}: 18.10 .76$

DM 150.- (incl. proceedings)

18-22 Oct.

Tucson, Ariz., USA

Annual Meeting of the Optical Society

J.W. Quinn, Executive Director, Optical Society of America, Suite 620,2000 L Street, N.W.,

Washington, D.C. 20036

Ab: 9.7 .76

US $\$ 40$. -

20-22 Oct.

New Orleans, USA

Scintillation and Semiconductor Counter Symposium

D.C. Cook, Code 6603C, Naval Research

Laboratory, Washington, DC 20375

25-27 Oct.

Denton, Texas, USA

Small Accelerators in Research. Teaching and Industrial Applications

Jerome L. Duggan, Physics Department, North Texas University, Denton, TX 76203

28-30 Oct.

East Lansing, Mich., USA

* Meeting of the Nuclear Physics Division (APS)

31 Oct. - 3 Nov.

San-Francisco, USA

Meeting of the American Ceramic Society

S.K. Dutta, Attn: DRXMR-EO, Army Materials and Mechanics Research Center, Arsenal Street. Watertown, Mass. 02172

2 Nov.

Cranfield, Bedford, UK

Time-Dependent Stresses and Strain in Plastic Materials

The Meetings Officer, The Institute of Physics, 47. Belgrave Square, London SW1X $8 Q X$ Ab: 30.4 .76

8-12 Nov.

Troy, N.Y., USA

* 4th Workshop: Laser Interaction and Related Plasma Phenomena

9-10 Nov.

London, UK

Int. Symposium on Techniques for Retrieval of Chemical Information

John F. Gibson, The Chemical Society, Burlington House, London W1V OBN

9-12 Nov.

London, UK

* Millimetric Waveguide Systems

14-18 Nov.

San Francisco, Cal., USA

* Meeting of the Plasma Physics Division (APS)

14-19 Nov.

Chicago, USA

Joint Meeting of American Association of Physicists in Medicine and Radiological Society of North America

Lawrence Lanzl, Box 420, Franklin McLean Memorial Research Institute, 950 E. 59th Str. Chicago, IL 60637

15-19 Nov.

Philadelphia, USA

3rd Annual Meeting of Federation of Analytical Chemistry and Spectroscopy Societies

E.G. Brame Jr., Du Pont Experimental Station, Wilmington, Delaware 19898

15-19 Nov.

* Hadron Structure '76 
19 Nov.

Wembley, UK

$X$-Ray Diffraction

The Meetings Officer, The Institute of Physics, 47, Belgrave Square, London SW1X $8 \mathrm{QX}$ Ab: 1.10 .76

22-24 Nov.

Eugene, Oregon, USA * Meeting of the Fluid Dynamics Division (APS)

22-25 Nov.

London, UK

* The Future of Aircraft All-Weather Operations?

29 Nov. - 4 Dec.

Paris, France

* Congrès National de Physique des Plasmas

1-3 Dec.

Annapolis, Md., USA

Topical Meeting on Optical Phenomena in Infrared Materials

Optical Society of America, Optical Phenomena

in Infrared Materials, Suite 620,2000 L Str. N.W., Washington, D.C. 20036 ,

Ab: 3.9 .76

US $\$ 50$. -

5-8 Dec.

Washington, D.C., USA

* International Electron Devices Meeting

6-8 Dec.

Lincoln, Nebraska, USA

* Electron and Atomic Physics Div. Meeting (APS)

6-10 Dec.

San Juan, Puerto-Rico

Submillimeter Waves and their Applications

J.J. Gallagher, Georgia Institute of Technology,

Atlanta, Ga. 30332

Ab: 2.8 .76

6-10 Dec.

Paris, France

Plasma Physics Conference

Mr. Fitaire, Laboratoire de Physique des Plasmas, Bâtiment 212, F-9145 Orsay

13-14 Dec.

London, UK

Newer Aspects of Molecular Relaxation Processes

Mrs. Y.A. Fish, Faraday Division, The Chemica Society, Burlington House, London W1V OBN

A: Nov, 76 / P: $31.8 .76 / 150$

13-15 Dec.

Williamsburg, Va., USA

Atmospheric Aerosols, their Optical Properties and Effects

Optical Society of America, 2000 L Street, N.W. Washington, D.C. 20036

P: 10.9 .76

US $\$ 50$ -

15-18 Dec.

Erice, Italy

Round Table: Media and Information Revolution

Main Secretariat, EMCSC, Via Guarnotta 26 , I-91016 Erice

19-22 Dec.

Erice, Italy

Round Table: Should Europe have an Institute for Solar Energy Research ?

Main Secretariat, EMCSC, Via Guarnotta 26, I-91016 Erice

20-22 Dec.

Stanford, Cal., USA

* General Meeting of American Physical Society

27 Dec. - 1 Jan. 1977

Calcutta, India

Advanced Mathematical Techniques in Physical Sciences

M. Dutta, Centre of Advanced Study in Applied Mathematics, 92 Acharya Prafulla Chandra Road, Calcutta - 700009

Ab: 18.9 .76

\section{7}

5-7 Jan.

Manchester, UK

14th Annual Solid State Physics Conference

The Meetings Officer, The Institute of Physics, 47. Belgrave Square, London SW1X 8QX
14-17 Feb.

Sao Paulo, Brazi

5th Int. Conf. on Luminescence Dosimetry

Watanabe, IEA, Cidade Universitaria Pinheiros-Sao Paulo

Ab: 1.10 .76

15-17 Feb.

Prague, Czechoslovakia

4th Int. Conf. on Measuring Systems -

EMISCON 77

Secretariat EMISCON 77, O. Michalcova, Gorkého nam. 23, 11282 Prague 1

A: $15.6 .76 / A b: 15.6 .76$

English, Czech, Slovak, Russian, German

16-18 Feb.

Philadelphia, USA

International Solid State Circuits Conference

Technical Activity Board, IEEE Inc., 345 E. 47th 1200 Street, New York, N.Y. 10017

21-23 Feb.

London, UK

Int. Conf. on Reliability of Power Supply Systems

Conference Department, IEE, Savoy Place, London WC2R OBL

$A b: 12.4 .76 / P: 1.9 .76$

21-25 Feb.

Asilomar, Cal., USA

Meeting of the American Crystallographic

Association

M.H. Mueller, ACA Secretary, Argonne National Laboratory, Building 212, Argonne, III. 60439

22-24 Feb.

Williamsburg, Va., USA

Optical Fiber Transmission II

Optical Society of America, 2000 L Street, N.W. Washington, D.C. 20036

P: 19.11 .76

8-10 March

London, UK

Use of Electricity for Materials Processing and Conservation

IEE Conference Department, Savoy Place. London WC2R OBL

Ab: $12.4 .76 /$ P: 10.9 .76

\section{6-18 March}

Chicago, III., USA

Particle Accelerator Conference

Technical Activities Board, IEEE,

345 E. 47th Street, New York, N.Y. 10017

\section{3-25 March}

Guildford, UK

Nuclear Structure

The Meetings Officer, The Institute of Physics, 47, Belgrave Square, London SW1X $8 Q X$

23-25 March Leicester, UK

Radiation Effects in Liquids and Solids

M.C.R. Symons, Department of Chemistry, The University, Leicester LE1 7RH

A: Feb. $77 / \mathrm{Ab}: 31.5 .76 / \mathrm{P}: 1.12 .76 / 150 /$ inv,

27-30 March Coventry, UK

3rd Interdisciplinary Surface Science Conference

D.P. Woodruff, Physics Dept., University of

Warwick, Coventry CV4 7AL

28-31 March

Darmstadt, FRG

Macromolecules and Organic Compounds under High Pressure: Chemistry and Physics

G. Luft, Institut für Chemische Technologie,

Technische Hochschule, Petersenstr. 15,

D-61 Darmstadt

A: $15.1 .77 /$ P: $15.1 .77 / 200$

English, German, French

29-31 March

Weybridge, UK

๑) Luminescence Processes in Phosphors for Cathode-Ray Tubes and Lamps

Paul Greenough, The General Electric Co. Ltd., Hirst Research Centre, Wembley, Middlesex, HA9 7PP

$70 /$ inv.
30 March - 1 April

London, UK

Pair Interactions in Molecules

J.F. Gibson, The Chemical Society, Burlington House, London W1V OBN

A: Feb. 77

5-6 April

Magnetic Resonance in Conductors

J.H. Strange, Hon. Sec., British Radio

Spectroscopy Group, Physics Laboratory, The

University, Canterbury, Kent

17-22 April

Noordwijkerhout, NL 6 th Int. Conf. on Molecular Beams

A.E. de Vries, FOM-Institute for Atomic and

Molecular Physics, Kruislaan 407, Amsterdam

Ab: $1.9 .76 / P: 1.2 .77$

hFI. 180.-

18-22 April

Grenoble, France

3e Congrès International de l'Electrostatique

René F. Challande, Commission Electrostatique,

Association Nationale de la Recherche

Technique, 44, rue Copernic, 75116 Paris

19-21 Apri

New York, USA

Electro/77

W.C. Weber, Jr., Electro, 999 N. Sepulveda Blvd El Segundo, Cal. 90245

P: 22.7 .76

24-30 April Paris, France

4th Int. Congress of the International Radiation Protection Association

Gilbert Bresson, General Secretary, IRPA,

B.P. 33, F-92260 Fontenay-aux-Roses

\section{5-27 April}

Phœnix, Ariz., USA

Circuits and Systems

W.G. Howard, Motorola Integ. Circuits Ctr., MS MR, POB 20906, Phœnix, Ariz. 85036

21-27 May

Banff Springs, Alberta, Canada

6th Int. Symposium on Magnetic Resonance

R.W. Dolan, c/o National Research Council of

Canada, Ottawa, Canada K1A ORA

English, French

23-25 May

Plasma Science

R.P. Hickok, Rensselaer Polytechnic Institute, Troy, N.Y. 12181

$A b: 2.1 .77 / 200$

23-27 May

London, UK

Electricity Distribution (CIRED 1977)

IEE Conference Department, Savoy Place, London WC2R OBL

\section{Summer}

Sweden

5th Int. Cont. on Chemical Thermodynamics

Prof. Sunner, Thermochemistry Laboratory, Lund IUPAC

5-10 June

Los Angeles, USA

International Magnetics Conference (INTERMAG)

Geoffrey Bate, IBM Corporation, P.O. Box 1900, Boulder, Co. 80302

6-10 June

Planetary Nebulae

Ithaca, N.Y., USA

Y. Terzian, Center for Radiophysics and Space Research, Space Science Building, Cornell University, Ithaca, N.Y. 14850 $150 /$ inv.

IAU

12-17 June

New York, USA

Meeting of the American Nuclear Society

David Pettengill, American Nuclear Society, 244 East Ogden Avenue, Hinsdale, III. 60521

19 June - 1 July

London, UK

Ultrasonics International

Z. Novak, Conference Organizer, IPC House, 32 High Street, Guildford, Surrey GU1 3EW

Ab: $31.12 .76 / P: 1.6 .77$ 
20-23 June

Saskatoon, Canada

1977 Congress of Canadian Association of Physicists

Canadian Association of Physicists, Suite 903, 151 Slater Street, Ottawa K1P $5 \mathrm{H}_{3}$

21-24 June

Berlin-West

Matrix Isolation Spectroscopy

H. Gerischer, Fritz-Haber-Institut, Faradayweg 4-6, D-1000 Berlin 33

Ab: $31.12 .76 / P: 15.4 .77 / 120$

English / DM 80.-

27 June -1 July

Palaiseau, France

3rd Int. Congress on Waves and Instabilities in Plasmas

T.D. Mantel, Laboratoire P.M.I. Ecole

Polytechnique F-91120 Palaiseau

A: 29.4 .77 / P: $29.4 .77 / 400$

English / FF 200.-

27 June - 1 July

7th Int. Conf. on Amorphous and

Semiconductors

Conference Secretariat, Centre for Industria

Consultancy and Liaison. University of

Edinburgh, 14 George Square, Edinburgh EH8 9JZ

IUPAP

5-7 July

Durham, UK

Rare Earths and Actinides

The Meetings Officer. The Institute of Physics, 47. Belgrave Square, London SW $1 \mathrm{X} 8 \mathrm{QX}$ A: 1.6.77/P: $1.3 .77 / 120 /$ inv

\section{2-15 July}

University, Reymonta 4, PL 30-059 Cracow 16

17-22 July

Cambridge, Mass., USA

5th Int. Conf. on Crystal Growth

K. Nassau, Bell Laboratories, Murray Hill, N.J. 07974

IUCr, IUPAC

21-27 July

Paris, France

10th Int. Conf. of Physics, Electronic and Atomic Collisions (ICPEAC)

Michel Barat, Laboratoire des Collisions

Atomiques, Bât. 220, Université Paris-Sud, F-91405 Orsay Cedex

26-28 July

Electromagnetic Compatibility

Seattle, USA

B.L. Carlson, Jr., The Boeing Co., РОВ 3029 Seattle, WA 90004

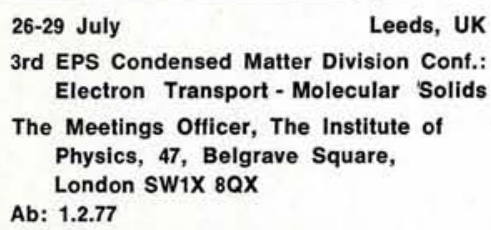

\section{3-26 Aug.}

Plovdiv, Bulgaria

15th International Cosmic Ray Conference

B. Betev, Institute for Nuclear Research and

Nuclear Energy, 72 Blvd. Lenin, Sofia 1113

Ab: 25.1 .77 / P: $1.5 .77 / 500 /$ inv.

English / IUPAP / US \$ 60.- (incl. Conf. papers)

15-18 Aug.

Haifa, Israel

Magnetic Alloys and Oxides

R.A. Buckwald, Dept. of Physics, Technion, Israel 200

Institute of Technology, Haifa 32000

\section{2-25 Aug.}

Prague, Czechoslovakia

Int. Conf. on Technical Diagnostics

Sekretariat, Int. Conf. Technical Diagnostics, Dum

techniky CVTS Praha, Gorkého nám. 23 ,

111282 Prague 1

Ab: 158.76

English, Czech, Slovak, Russian 13th IUPAP Conf. on Statistical Physics

I. Riess, Physics Department, Technion, Haifa IUPAP

29-31 Aug.

Dublin, Ireland

3rd Specialized Colloque Ampère: Optical Studies in Magnetic Resonance Spectroscopy

B. Henderson, Physical Laboratory, Trinity College, Dublin 2

£ 20.-

30 Aug. - 2 Sept.

Tallinn, USSR

The Structure of the Universe and the Formation of Galaxies

J. Einasto, W. Struve Astrophysical Observatory, Tartu, Toravere, Estonia, USSR

(IAU, under consideration)

30 Aug. - 3 Sept.

Oxford, UK

4th European Crystallographic Meeting

C.K. Prout, Chemical Crystallography Laboratory, 9 Parks Road, Oxford OX1 3QS

30 Aug. - 7 Sept. Prague, Czechoslovakia 20th Colloquium Spectroscopicum Internationale and 7 th Int. Conf. on Atomic Spectroscopy

Vaclav Sychra, VSCHT, Suchbatarova 1905 16628 Prague 6

Ab: 15.10 .76

English, Russian, German, French

\section{September}

Hertzian Optics and Dielectrics

G. Raoult, B.P. 45, F-63170 Aubière

50

English, French

September

Bamberg, FRG

The Interaction of Variable Stars with their Environment

R. Kippenhahn, Institut für Astrophysik, MaxPlanck-Institut für Physik und Astrophysik Föhringer Ring 6, D-8 Munich 40 $50 /$ inv.

IAU

5-9 Sept.

Montpellier, France

5th Int. Conf. on Vacuum UV Radiation Physics

Mme N. Pouey, CNRS, Laboratoire de Bellevue,

1, Place Aristide-Briand, F-92190 Meudon

5-10 Sept.

Paris, France

International Conference on Lattice Dynamics

M. Balkanski, Laboratoire de Physique des Solides,

Tour $13,2 e$ étage, 4 place Jussieu,

F-75230 Paris Cedex 05

A: 15.6.77 / Ab: 15.3 .77 / P: 1.10.77

1000

US $\$ 80 .-$

12-16 Sept.

Vienna, Austria

7th Int. Vacuum Congress and 3rd Int. Conf. on Solid Surfaces

R. Dobrozemsky, 7th IVC and 3rd ICSS, Postfach 300, A-1082 Vienna

Ab: $1.3 .77 / 1200$

IUVSTA

English, French, German

12-17 Sept.

Berlin, GDR

13th Int. Conf. on Phenomena in Ionized Gases

G. Hinzpeter, Academy of Science GDR, POB 73, 108 Berlin DDR

\section{3-15 Sept.}

Oxford, UK

Ion-Ion and Ion-Solvent Interaction

A.K. Covington, Physical Chemistry Dept., The University, Newcastle upon Tyne NE1 7RU

A: $1.8 .77 / \mathrm{Ab}: 30.9 .76 / \mathrm{P}: 1.5 .77 / 150$

18-23 Sept.

Leningrad, USSR

4 th Int. Meeting on Ferroelectricity (IMF 4)

G.A. Smolensky, A.F. Ioffe, Physico-Technica

Institute, Academy of Sciences of the USSR

Polytechnicheskeja, 194021 Leningrad 26
19-22 Sept.

London, UK

50th Anniversary of Discovery of Electron Diffraction

The Meetings Officer, The Institute of Physics 47. Belgrave Square, London SW1X $8 Q \mathrm{XX}$

22-26 Sept.

Istanbul, Turkey

10th World Energy Conference

E. Ruttley, Secretary-General, World Energy Conference, 5 Bury Street, St. James's London SW1Y $6 A B$, UK

26-29 Sept.

Varna, Bulgaria

6th Europhysics Conference on Macromolecular Physics - Phase Transitions in Bulk Polymers

M. Mihailov, EPS Conference, Central Laboratory for Polymers, Bulgarian Academy of Sciences 1113 Sofia

A: 1.1 .77 / Ab: $1.12 .76 / P: 1.6 .77 / 150 /$ inv.

SFr. 200.-

27-29 Sept.

London, UK

2nd Int. Conf. on Power Electronics - Powe Semiconductors and their Application

IEE Conference Department, Savoy Place. London WC2R OBL

October

Hvar, Yugoslavia

Photometry of Emission Line Objects

S. Kriz, Astronomical Institute of the Czechoslovak Academy of Sciences. 25165 Ondrejov, Czechoslovakia

(IAU, under consideration)

October

Budapest, Hungary

4th Symposium on Reliability in Electronics Relectronic '77

The Scientific Society for Telecommunication, Organizing Committee of Relectronic '77, P.O.Box $451 \mathrm{H}-1372$ Budapest

Ab: $31.5 .76 / P: 1.5 .77$

October

Upton, L.I., USA or Grenoble, F

3rd Int. Symposium on Neutron Capture Gamma Ray Spectroscopy and Related Topics

R.E. Chrien, Department of Physics, Brookhaven National Laboratory, Upton, L.I., N.Y. 11973

3-7 Oct.

Gatlinburg, Tenn., USA

4th Int. Conf. on Small-Angle Scattering of X-Rays and Neutrons

R.W. Hendricks, Metals and Ceramics Division, Oak Ridge National Laboratory, P.O. Box X, IUCr Oak Ridge, TN 37830

4-8 Oct.

Bordeaux, France

1st Int. Meeting on New Sources of Energy

Jean-Loup Gallien, Chargé des Relations Extérieures, TECHNOEXPO, 8, rue de 1 Michodière, F-75002 Paris

27-29 Oct.

Rochester, N.Y, USA Meeting of the Nuclear Physics Division (APS) American Physical Society, 335 E. 45th Street, New York, N.Y. 1001

7-11 Nov.

Atlanta, Georgia, USA

Meeting of the Plasma Physics Division (APS)

American Physical Society, 335 E. 45th Street, New York, N.Y. 10017

8-11 Nov.

Minneapolis, USA

Magnetism and Magnetic Materials

E.J. Torok, Univac Division, Sperry Rand, POB 3252, St. Paul, MN 55116

21-23 Nov.

Miami Beach, USA

General Meeting of the American Physical Society

American Physical Society, 335 E. 45th Street, New York, N.Y, 10017 
27 Nov. - 2 Dec.

San Francisco, USA

Meeting of the American Nuclear Society

David G. Pettengill, American Nuclear Society,

244 East Ogden Avenue, Hinsdale, III. 60521

5-7 Dec.

Knoxville, Tenn., USA

Electron and Atomic Physics Div. Meeting (APS) American Physical Society, 335 E. 45th Street, New York, N.Y. 10017

13-14 Dec.

Oxford, UK

Electrocrystallization, Nucleation and Phase Formation

A. Bewick, Department of Chemistry, The University, Southampton $\mathrm{SO} 5 \mathrm{NH}$

A: Nov. 77 / P: Aug. $77 / 150$

\section{8}

15-17 Feb. Philadelphia, USA

Int. Solid State Circuits Conference

Technical Activities Board, IEEE Inc., 345 E. 47th Street, New York, N.Y. 10017

March

Toulouse, France

2nd European Conference on Solar Physics

A.D. Fokker, Astronomical Institute, Zonnenburg 2.

Utrecht, The Netherlands 300

\section{April}

Florence, Italy

International Magnetics Conference (INTERMAG) Joe Suozzi, Bell Telephone Labs. Inc., Whippany,

N.J. 07981, USA

Ottawa, Canada

16-19 May

surements

A.F. Dunn, Nationch Montreal Road, Ottawa, Ontario

3-7 July

York, UK

4th International Meeting on Nuclear Magnetic Resonance Spectroscopy

John F. Gibson, The Chemical Society,

Burlington House, London W1V OBN

31 July - 9 Aug.

Toronto, Canada

9th Int. Meeting on Electron Microscopy

G.T. Simon, Chairman, Organising Committee

Dept. of Pathology, Bansting Institute,

100 College Street, Toronto, Ont. M5G 1L5

\section{5-29 Sept. \\ York, UK \\ Fourth EPS General Conference \\ Provisional information: EPS Secretariat,}

P.O.Box 39, CH-1213 Petit-Lancy 2

18-20 Oct.

Washington, D.C., USA

Nuclear Science Symposium

Scintillation and Semiconductor Symposium

Nuclear Power Systems Symposium

Louis Costrell, US Department of Commerce,

National Bureau of Standards, Washington DC 20234

30 Oct. -4 Nov.

Meeting of Plasma Physics Division (APS)

\section{USA}

American Physical Society, 335 E. 45th Street,

New York, N.Y. 10017

14-17 Nov.

Cleveland, Ohio, USA

Magnetism and Magnetic Materials

J.B. Blade, R \& D Center, Addressograph-

Multigraph, 19701 South Mile Road,

Warrenville Heights, $\mathrm{OH} 44128$

\section{Ecole Polytechnique Fédérale de Lausanne}

CENTRE DE RECHERCHES

\section{EN PHYSIQUE DES PLASMAS}

\section{Research Associates}

The CRPP is forming new research groups in the fields of toroidal confinement and laser plasma interactions.

The CRPP offers appointments for highly qualified physicists in the field of experimental and theoretica plasma physics and instrumentation.

Appointments will be made for two years with possible extensions for further two year terms.

Applicants are expected to have earned their $\mathrm{PhD}$ degree in plasma physics, astrophysics, microwave engineering or optics within the past ten years.

Application forms may be obtained by writing to N. Marendaz CRPP, 21, avenue des Bains, 1007 Lausanne, Switzerland. The applicant should request three persons to whom he is well known to send letters of recommendation.

\section{Schools}

\section{6}

28 June -7 July Erice, Italy

Fast Transient Phenomena in Astrophysics

G. Sironi, Laboratorio di Fisica Cosmica, CNR Istituto di Fisica, V. Celoria 16, I-20133 Milan A: 15.5 .76

SFr. $700 .-$

28 June - 10 July Varenna, Italy * Isolated Gravitating Systems in General Relativity

1-30 July

La Rochelle, France * Summerschool of Space Physics - High Energy Astrophysics

5-16 July Abingdon, Oxfordshire, UK

* 1976 Culham Plasma Physics Summerschool: Basic Plasma Physics - Modern Developments and Applications

5 July - 14 Aug.

Les Houches, France Weak and Electromagnetic Interactions at High Energy

Ecole d'Eté de Physique Théorique,

F-74310 Les Houches

A: $10.3 .76 / 40$ / inv.

FF 1800.- (all incl.)

7 July - 19 Aug Trieste, Italy

* Teaching of Physics at Tertiary Level

\section{2-24 July}

Metrology and Fundamental Constants

S. Leschiutta, Istituto Elettrotecnico Nazionale

"G. Ferraris", Corso Massimo D'Azeglio 42, I-10125 Turin

A: $30.4 .76 / 75 /$ inv.

English, French

12-30 July

Caracas, Venezuela

* XVI. Latin American School of Physics
12-31 July

Cargèse, France

Developments in Quantum Field

Field Theory and Statistical Mechanics

Mile M.-F. Hanseler, Secretary Ecole Cargèse,

L.P.T.H.E., Tour 16, Univ, P. et M. Curie,

4 place Jussieu, F-75230 Paris Cedex 05

23 July - 8 Aug.

Erice, Italy

Understanding the Fundamental Constituents of Matter

Miss M. Zaini, Int. School of Subnuclear Physics,

CERN, CH-1211 Geneva 23

A: 30.6 .76

SFr. 1000.-

26 July - 6 Aug.

Capri, Italy

* Photon Correlation Spectroscopy and

Velocimetry

26 July - 7 Aug.

Varenna, Italy

* Elementary Modes of Excitation in Nucle

1-21 Aug.

St. Andrews, UK

* Scottish Universities Summer School in Physic

1976 - Fundamentals of Quark Models

1-29 Aug.

Uppsala, S and Dalseter, $N$

* Quantum Chemistry and Solid State Physics

1 Aug. - 30 Sept.

Orsay, France

* X-Ray and UV Spectra of Hot and Dense Plasmas

1 Aug. - 30 Sept.

Orsay, France

* Plasmaphysics Applied to Active Phenomena of the Sun

2-13 Aug.

Stanford, Cal., USA

SLAC Summer Institute on Particle Physics Weak Interactions at High Energies and the Production of New Particles

M. Zipf, SLAC, P.O. Box 4349, Stanford CA 94305

A: 30.4 .76

2-20 Aug.

Nathiagali, Pakistan

* Summer College on Physics and Contemporary Needs

8-21 Aug.

Erice, Italy

The Sun, a Tool for Stellar Physics

M. Rigutti, Osservatorio Astronomico, Via

Moiariello 16, I-80131 Naples

A: 30.6 .76

US $\$ 250 .-$

9-21 Aug.

Nijenrode Castle, N

High-Energy Chemical Spectroscopy and Photochemistry

H.H. Brongersma, Philips Research Labs. Eindhoven

16-27 Aug.

Istanbul, Turke

Two Phase Flows and Heat Transfer

S. Kakaç, Middle East Technical University (O.D.T.O), Ankara

17-29 Aug.

Bolzano, Italy

* Chemistry and Physics of One-Dimensional Metals

22 Aug. - 3 Sept.

Banff, Alberta, Canada

Critical Phenomena and Phase Transitions

D.D. Betts, Theoretical Physics Inst., University, Edmonton, Alberta, Canada

23 Aug. - 4 Sept.

Bielefeld, FRG

International Summer Institute for Theoretical Physics - Many Degrees of Freedom in Particle Physics and Field Theory

Mrs, A. Baker, ZIF, Universität Bielefeld, Wellenberg 1, D-4800 Bielefeld 1

* For full details please see March issue of Europhysics News 
24 Aug. - 3 Sept.

Herceg-Novi, Yugoslavia

* Radiation Protection: Current Problems and Concerns of the Health Physicist

25 Aug. - 5 Sept.

Dubrovnik, Yugoslavia

2nd Adriatic Meeting on Particle Physics Structure of Hadrons

N. Zovko, Ruder Boskovic Institute, P.O. Box 1016, YU-41001 Zagreb

A: 31.5 .76

26 Aug. -4 Sept.

Predeal, Romania

* Int. School on Nuclear Physics - Heavy Ion Physics

29 Aug. - 11 Sept. Masurian Lakes, Mikolajki, PL * 9th Summer School in Nuclear Physics

29 Aug. - 12 Sept.

Corsica, France

Material Characterization using Ion Beams

J.P. Thomas, Institut de Physique Nucléaire,

43. Boulevard du 11 Novembre 1918,

F-69621 Villeurbanne

30 Aug. - 11 Sept.

Ghent, Belgium

* Electrons in Finite and Infinite Structures

31 Aug. - 10 Sept.

Alghero, Sardinia,

3rd Course on Solar Energy Conversion

G. Giaquinta, Istituto di Fisica, Corso Italia, 57, I-95129 Catania

1 Sept. - 1 July 1977

16th Int. Seminar on Physics and

7th Int. Seminar on Chemistry

International Seminars, University of Uppsala,

Box 530, S-751 21 Uppsala

1-10 Sept.

Gardone Riviera, Italy

Small Scale Applications of Superconductivity

S. Foner and B.B. Schwartz, Codirectors,

Francis Bitter National Magnet Laboratory,

MIT, 170 Albany Street, NW14, Cambridge,

Mass. 02139, USA

A: $1.6 .76 / 70 /$ inv.

1-15 Sept.

Erice, Italy

Tachyons and Related Topics

R. Mignani, Istituto Fisica Teorica, Università Piazzale delle Scienze 5, 1-00185 Rome

A: 31.7 .76

SFr. 700.-

1-15 Sept.

Villa le Pianore, Tuscany,

Coherent Optical Engineering

F.T. Arecchi, C.I.S.E., C.P. 3986, 1-20100 Milan

A: 30.6 .76

US $\$ 300,-$

2-14 Sept.

Erice, Italy

The Mesonic Interface between Nuclear Structure and Particle Physics

Sir Denys Wilkinson, Nuclear Physics Lab., Keble Road, Oxford, UK

A: 15.8 .76

US $\$ 250 .-$

5-18 Sept.

Altavilla Milicia, Sicily,

T. Axenrod, Dept. of Chemistry. The City College of the City University, New York, N.Y. 10031, USA

6-10 Sept.

Montpellier, France

* Probability and Statistics in Teaching of Physics in Secondary Schools

Introductory Teaching of Physics in Early Years of Secondary Schools

6-17 Sept.

Varenna, Italy

* 3rd Symposium on Plasma Heating in Toroidal Devices

* For full details please see March issue of Europhysics News
Perpignan, France

* Ecole d'Eté sur les Transferts d'Energie par Rayonnement Thermique

6-19 Sept.

Honolulu, Hawaii, USA

Deep Underwater Muon and Neutrino Detection 1976 Summer Workshop

DUMAND Summer Workshop, Fermi Nationa Accelerator Laboratory, P.O. Box 500 Batavia, IL 60510

9-23 Sept.

Modern Methods in Vibrational Spectroscopy

W.J. Orville-Thomas, Dept. of Chemistry and Applied Chemistry, University, Salford M5 4WT, UK

12-17 Sept.

Birmingham, UK

Course on Thermal Neutron Scattering

D.K. Ross, Department of Physics, University of Birmingham, P.O. Box 363, Birmingham B15 2TT

12-24 Sept.

Alghero, Sardinia,

Synchrotron Radiation Research

G. Giaquinta, Istituto di Fisica, Corso Italia, 57, 1-95129 Catania

A: 31.5 .76

12-25 Sept. La Colle-sur-Loup, near Nice, F * 1976 CERN School of Computing

13-23 Sept. Pula, Yugoslavia

Magnetic Resonance in Condensed Matter. Recent Developments

Gojmir Lahajnar, Scientific Secretary, J. Stefan Institute, University of Ljubljana, Jamova 39 , POB 199/IV, YU - 61001 Ljubljana

A: $31.5 .76 / 100$

US $\$ 50$ -

13-24 Sept.

Edinburgh, UK

Course on Phonons and Spectroscopy (especially Raman Spectroscopy) of Crystals

R.F. Ferrier, Department of Natural Philosophy, Glasgow University, Glasgow, Scotland

14-24 Sept.

Maria-Laach (Eiffel) FRG

Herbstschule für Hochenergiephysik: Physik mit Elektron-Positron Speicherringen und Neutrino Reaktionen

J.K. Bienlein, DESY, Notkestieg 1

D-2000 Hamburg 52

A: $30.6 .76 / 50$

German / DM 200.-

19 Sept. - 1 Oct.

Erice, Italy

kamak Reactors for Breakeven

Knoepfel, Centro Gas Ionizzati, C.P. 65, I-00044 Frascati

A: $15.7 .76 / 70$

US $\$ 260$. -

19 Sept. - 3 Oct.

Basko Polje, Yugoslavia

International School of Elementary Particle Physics - 12th Session

H. Braun, Maître de Recherche, Div. des Hautes Energies, C.B.H., Centre de Recherches Nucléaires, 23 rue du Loess,

F-67037 Strasbourg - Cedex 80

A: 1.5 .76

SFr. 650 -

22 Sept. - 10 Dec.

Trieste, Italy

Applications of Analysis to Mechanics

P. Budini, International Centre for Theoretica

Physics, Miramare, РОB 586, I-34100 Trieste A: 31.5 .76

Ochool on Crystal Growth

G. Schmidt, Martin Luther Universität, Sektion Physik, DDR - 402 Halle

16-31 Oct.

Erice, Italy

Energy Demand and Use

F. Amman, Istituto di Fisica Applicata,

Via Bassi, 6, 1-27100 Pavia

A: 31.8 .76

US $\$ 270$. -

10-22 Nov.

Erice, Italy

Theoretical Aspects of the Behaviour of Beams in Accelerators and Storage Rings

K. Johnsen, ISR Division, CERN

$\mathrm{CH}-1211$ Geneva 23, Switzerland

A: 1.9 .76

SFr. $750 .-$

\section{3-30 Nov.}

Erice, Italy

Imaging and Displaying Devices

C. Castagnoli, Laboratorio di Cosmogeofisica, Corso Fiume 4, 10125 Turin

24 Nov. - 5 Dec.

Erice, Italy

Black Holes, Magnetosphere and Vacuum Polarization

R. Ruffini, Princeton University, Dept. of Physics, POB 708 , Princeton, N.J. 08540, USA

\section{7}

26 Jan. - 18 March

Trieste, Italy

Winter College on Atomic and Molecular Physics International Centre for Theoretical Physics, P.O. Box 586, 1-34100 Trieste

A: 15.6 .76

18-28 April

Rehovot, Israel

Bat-Sheva Seminar on Electron Density Mapping in Molecules and Crystals

F.L. Hirshfeld, Department of Structural

Chemistry, Weizmann Institute of Science. Rehovot

10-16 July

Durham, N.H., USA

3rd International Summer School on Crystal

Growth

K. Nassau, Bell Laboratories, Murray Hill, IUC N.J. 07974

1-17 Sept.

Varenna, Italy

Theory of Magnetically Confined Plasma

E. Sindoni, Laboratorio di Fisica del Plasma, Istituto di Fisica, Via Celoria, 16,

I-20133 Milan

\section{Editor: E.N. Shaw}

Meetings Compilation: S. Newman

Editorial Board:

G.J. Béné, B. Glovannini, M. Guenin,

B. Hauck, J. Muller, S. Newman

All correspondence to:

Editor, EUROPHYSICS NEWS

European Physical Society,

P.O. Box 39

CH - 1213 Petit-Lancy 2, Switzerland

Phone: Geneva 931132

Published by the European Physical Society

Printed by: Ed. Cherix et Filanosa SA

$\mathrm{CH}-1260$ Nyon, Switzerland 